\title{
变速变排量液压驱动系统的动态过程能效优化
}

\author{
黄海鸿 $^{1,2}$ 唐运先 ${ }^{1,2}$ 金 瑞 ${ }^{1,2}$ 李 䂞 ${ }^{1,2}$ 李大成 ${ }^{3}$ 刘志峰 $^{1,2}$ \\ (1. 合肥工业大学机械工程学院 合肥 230009;
}

2. 机械工业绿色设计与制造重点实验室 合肥 230009;

3. 安徽源典科技有限公司 合肥 230051)

\begin{abstract}
摘要: 液压系统广泛应用于工程机械与液压成形装备等领域, 但液压驱动单元输出功率与负载需求功率不匹配造成的低能效 问题一直不可忽视。变速变排量驱动单元可协调控制电动机转速和泵排量, 实现液压系统在各稳定工况下驱动单元输出功率 与负载需求功率的匹配, 提高液压系统能量效率。为进一步提高变速变排量驱动单元在复杂多变工况下的能效, 对其动态过 程中的能效进行深入研究。建立了动态过程能耗优化模型, 通过优化模型并结合试验分析得出动态过程最小能耗对应的电动 机加速度。通过试验研究了不同加速度下的系统稳定性, 分析得出液压缸推杆振动与电动机转速波动有关, 可通过调整电动 机加速度以限制电动机转速波动从而保证系统稳定性。最后, 以液压拉深成形过程为例, 在保证系统稳定性的前提下分析拉 深成形过程的动态能耗, 当电动机采用最佳加速度时, 整个动态过程的能效平均提高了 $8.0 \%$ 、转速波动量平均降低了 $14.9 \%$ 。
\end{abstract} 关键词: 功率匹配; 动态过程; 能效; 稳定性; 电动机加速度

中图分类号: TH137

\section{Energy Efficiency Optimized in the Dynamic Process of a Variable-speed Variable-displacement Pump Unit}

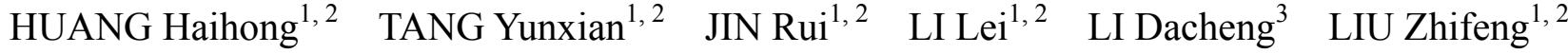

(1. School of Mechanical Engineering, Hefei University of Technology, Hefei 230009;

2. Key Laboratory of Green Design and Manufacturing for Machinery Industry, Hefei 230009;

3. Anhui Yuandian Technology Co., Ltd., Hefei 230051)

\begin{abstract}
Hydraulic systems are widely used in construction machinery and hydroforming equipment, but the low energy efficiency caused by the power mismatch between output power of the drive unit and demand power of the actuators cannot be ignored. By cooperatively adjusting the motor speed and pump displacement of a variable-speed variable-displacement pump unit, the power match degree can be improved under the stable operating conditions. In order to further improve energy efficiency of the variable-speed variable-displacement pump unit under complex and variable working conditions, energy consumption model of the drive unit during the dynamic process is established. The relationship between energy consumption and motor acceleration is obtained to acquire the optimum motor accelerations and the minimum energy consumption during dynamic process. Moreover, the stability with different motor accelerations of the hydraulic system is investigated by experiments, and the motor accelerations are selected according to the required working conditions for the preferable energy efficiency and stability. The results indicate that energy efficiency and speed fluctuation of the preferable motor acceleration can be improved by $8.0 \%$ and $14.9 \%$ respectively, compared with that of the default motor acceleration.
\end{abstract}

Key words: power matching; dynamic process; energy efficiency; stability; motor acceleration

\section{0 前言}

液压系统具有功率密度大、反应快和易控制

$*$ 国家自然科学基金资助项目(U20A20295，51722502)。20200405 收到 初稿, 20201204 收到修改稿
输出力(或力矩)等诸多优点, 在各领域中有着广 泛应用，但系统在运行过程中也存在较高的能量 损失。降低液压系统运行过程能量损失的方法主 要包括能量回收再利用和液压系统功率匹配。能 量回收再利用主要是利用蓄能器 ${ }^{[1]}$ 、飞轮 ${ }^{[2]}$ 和超 级电容 ${ }^{[3]}$ 等回收液压系统惯性能量并用于其他工 
作周期。此外, 一种新型双执行器节能系统直接 将其中一个执行器下降的重力势能转换用于另一 执行器上升的能量, 降低了能量转换次数, 实现 了能量回收的直接利用 ${ }^{[4]}$ 。

液压系统在运行过程中, 各工况负载需求功 率不同, 驱动单元的装机功率需要按照最高功率 配置, 造成了一些工况下负载需求功率与驱动单 元输出功率的不匹配。采用液压驱动单元分区控 制节能方法可提高液压系统驱动单元输出功率和 负载需求功率的匹配程度, 使驱动单元的输出功 率和该动作的消耗功率相匹配 ${ }^{[5]}$ 。随着变排量㫤 和变速电动机控制技术的发展, 很多学者采用变 排量洜或变速电动机调节液压驱动单元的输出流 量, 实现驱动单元的输出流量与执行器的需求流 量匹配 ${ }^{[6-7]}$ 。在对比变频电动机驱动定量洜、工频 电动机驱动变量百等驱动方式时, 发现成形过程 中变速电动机驱动变量㬌驱动方式更节能。

HUANG 等 ${ }^{[8]}$ 利用变速变排量液压驱动系统解决 了液压系统功率不匹配的问题, 并通过查表方式 得到各静态工况最佳效率对应的电动机转速和葲 排量, 但未考虑静态工况切换过程的动态能耗。 为降低变速变排量液压驱动系统的能耗, WILLKOMM 等以能耗最低为优化目标, 通过同 时控制电动机转速和洜排量两个变量, 得到注塑 过程各静态工况的电动机转速和百排量 ${ }^{[9]}$ 。针对 静态工况 (电动机转速和葲排量组合) 的切换过 程, WILLKOMM 等 ${ }^{[9]}$ 通过试验对比分析了不同 电动机加速度下的动态能耗特性, 但动态能量损 失与电动机加速度的理论关系仍不明晰。

变速变排量液压驱动单元具有较高的节能潜 力, 通过对电动机转速和洜排量的优化, 可在实 现液压系统输出功率与需求功率匹配的同时, 实 现驱动单元能效最优。但目前关于变速变排量液 压驱动系统在静态工况 (电动机转速和洜排量组 合)切换过程中的动态能耗研究较少。对于工况频 繁切换的液压设备, 如频繁升降的挖掘机动臂 ${ }^{[10]}$ 和频繁启停的装载机 ${ }^{[11]}$, 由于其负载一直变化, 在运行过程中存在大量动态切换过程。文献 [10] 中挖掘机一个工作周期为 $10 \sim 15 \mathrm{~s}$, 存在 5 个不 同工作阶段(回转、下降、挖掘、提升和卸料)、 4 次动态切换过程, 因此, 研究静态工况点频繁切 换的一类变速变排量液压驱动系统的动态能耗具 有理论意义与工程价值。

同时, 在变速变排量液压驱动系统的动态切
换过程中, 由于电动机转速波动造成的系统稳定 性问题也不可忽略 ${ }^{[12-13]}$ 。例如, 针对液压机等成 形装备, 电动机转速波动导致的液压系统不稳定 会造成工件的成形质量问题 ${ }^{[14-15]}$ 。目前, 针对运 行参数配置不当造成的液压系统稳定性问题, 主

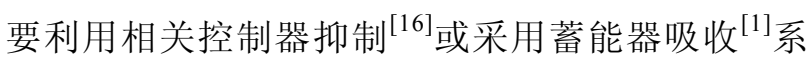
统产生的振动, 通过优化运行参数等主动减振手 段的研究较少 ${ }^{[14]}$ 。

本文针对工况频繁切换的变速变排量液压驱 动系统的动态过程, 基于能量流对驱动系统的动 态过程进行建模分析, 在保证系统稳定性的前提 下建立变速变排量液压驱动系统动态过程能耗优 化模型。搭建伺服电动机驱动比例变量洜试验平 台, 分析动态过程能量损失与电动机加速度的关 系, 验证理论模型的合理性; 通过试验分析电动 机加速度对系统稳定性的影响。最后以液压拉深 成形过程为例, 分析系统动态过程能耗优化模型 的效果。

\section{1 变速变排量驱动系统动态能耗模型}

\section{1 系统动态过程分析}

变速变排量液压驱动系统主要由变速电动机、 变量洜及相关驱动器组成。系统从电网获取电能, 由电动机转换成机械能, 再由洜转换成液压能, 从 而驱动液压设备工作。在液压系统运行过程中, 驱 动单元输出功率 $P_{\text {out }}$ 与负载需求功率 $P_{\text {req }}$ 的关系如 式(1)。

$$
P_{\text {req }}=F(t) \cdot v(t)=P_{\text {out }} \cdot \eta_{s}=p(t) \cdot Q(t) \cdot \eta_{s}
$$

式中, $p(t) 、 Q(t)$ 分别为驱动单元的输出压力、输出 流量; $\eta_{s}$ 为驱动单元与执行器间液压系统的能量效 率; $F(t)$ 和 $v(t)$ 分别是活塞所受负载力和运动速度。

实际上, 液压系统功率不匹配的主要原因是各 工况下液压驱动单元输出流量与执行器需求流量不 匹配。变速变排量液压驱动系统通过调整各工况下 的电动机转速和洜排量以实现流量匹配, 并通过对 每个静态工况的电动机转速和洜排量进行优化, 得 到所有工况驱动单元最佳效率下的电动机转速和原 排量组合 ${ }^{[8]}$ 。因此, 在变速变排量液压驱动系统运 行过程中, 就会存在工况 $i$ 下的电动机转速 $n_{i}$ 和原 排量 $V_{i}$ 组合向工况 $i+1$ 下的电动机转速 $n_{i+1}$ 和洜排 量 $V_{i+1}$ 组合切换的动态过程。变速变排量液压驱动 系统的动态过程如图 1 所示。 


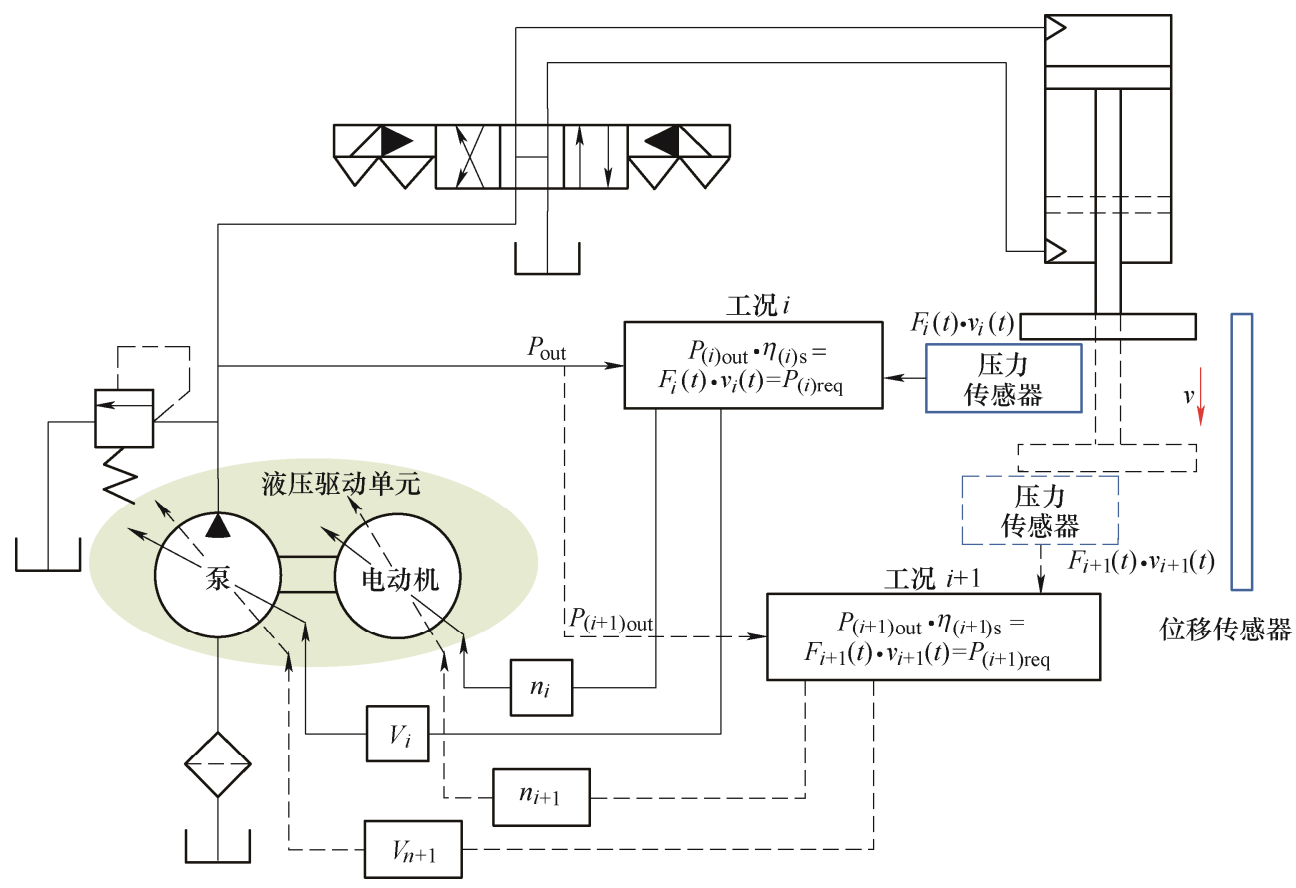

图 1 变速变排量液压驱动系统动态过程示意图

\section{2 系统动态能耗优化模型}

变速变排量液压驱动系统的动态过程是电动机 转速和泵排量组合从一个工况向另一个工况变化并 达到稳定的过程, 即工况切换过程。对于动态过程, 驱动单元的能量流及功率损失如图 2 所示。

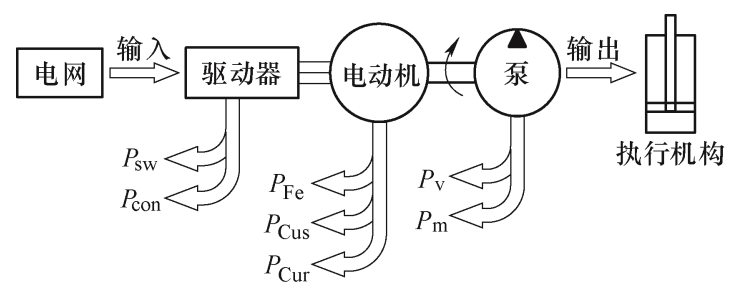

图 2 变速变排量驱动单元动态过程能量流及功率损失

根据能量流得电动机驱动器的功率损失 $P_{\mathrm{c}}$ 主 要包括通态损耗 $P_{\mathrm{con}}$ 和开关损耗 $P_{\mathrm{sw}}{ }^{[17]}$

$$
\begin{aligned}
& P_{\text {con }}=f\left(I_{\mathrm{s}}\right) \\
& P_{\mathrm{sw}}=g\left(I_{\mathrm{s}}\right)
\end{aligned}
$$

式中, $I_{\mathrm{s}}$ 为通过定子线圈的电流。

电动机的功率损失包括消耗于电动机定子绕组 的铁损 $P_{\mathrm{Fe}}$ 、定子铜损 $P_{\mathrm{Cus}}$ 、转子铜损 $P_{\mathrm{Cur}}$ 、机械损 耗和杂散损耗等。由于机械损耗和杂散损耗占总损 耗的比例较少, 一般分析中可忽略 ${ }^{[18]}$ 。

$$
\begin{gathered}
P_{\mathrm{Fe}}=K_{\mathrm{f}} \cdot n \\
P_{\mathrm{Cu}}=P_{\mathrm{Cus}}+P_{\mathrm{Cur}}=3\left(I_{\mathrm{s}}^{2}+I_{\mathrm{r}}^{2}\right) R
\end{gathered}
$$

式中, $K_{\mathrm{f}}$ 为常数; $I_{\mathrm{r}}$ 为转子电流; $R$ 为线圈电阻 ${ }^{[9]}$ 。

泵的功率损失包括容积损失 $P_{\mathrm{pv}}$ 和机械损失 $P_{\mathrm{pm}}$ 。其表达式如下 ${ }^{[8]}$

$$
P_{\mathrm{pv}}+P_{\mathrm{pm}}=\frac{\Delta p V n}{60}\left(\frac{1}{\eta_{\mathrm{pm}}}-\eta_{\mathrm{pv}}\right)
$$

式中, $\Delta p$ 为泵进出口压力差; $V$ 为原排量; $n$ 为电动 机转速; $\eta_{\mathrm{pm}}$ 和 $\eta_{\mathrm{pv}}$ 分别为百的容积效率和机械效率。 电动机的定子电流与励磁转矩的关系为

$$
I_{\mathrm{s}}=\frac{M_{\mathrm{e}}}{K_{2} \cdot \psi_{\delta}}
$$

式中, $M_{\mathrm{e}}$ 为电动机的励磁转矩; $K_{2}$ 为常数; $\psi_{\delta}$ 为气 隙磁链。

根据动力学原理可得到式(8)

$$
M_{\mathrm{e}}-M_{\mathrm{L}}=J_{\mathrm{t}} \cdot \alpha
$$

式中, $M_{\mathrm{L}}$ 为电动机受到的负载转矩; $J_{\mathrm{t}}$ 为换算至电 动机轴的等效转动惯量; $\alpha$ 为电动机的加速度。由 上述联立可得

$$
\begin{gathered}
I_{\mathrm{s}}=\frac{M_{\mathrm{L}}+J_{\mathrm{t}} \cdot \alpha}{K_{2} \cdot \psi_{\delta}} \\
P_{\mathrm{con}}=f \frac{M_{\mathrm{L}}+J_{\mathrm{t}} \cdot \alpha}{K_{2} \cdot \psi_{\delta}} \\
P_{\mathrm{sw}}=g \frac{M_{\mathrm{L}}+J_{\mathrm{t}} \cdot \alpha}{K_{2} \cdot \psi_{\delta}} \\
P_{\mathrm{Cu}}=3\left[\left(\frac{M_{\mathrm{L}}+J_{\mathrm{t}} \cdot \alpha}{K_{2} \cdot \psi_{\delta}}\right)^{2}+I_{\mathrm{r}}^{2}\right] R
\end{gathered}
$$

联立式(2) (12)可得驱动单元动态过程的功率 损失 $P$

$$
\begin{aligned}
& P=P_{\mathrm{con}}+P_{\mathrm{sw}}+P_{\mathrm{Cu}}+P_{\mathrm{Fe}}+P_{\mathrm{pv}}+P_{\mathrm{pm}}= \\
& \varphi\left(M_{\mathrm{L}}, J_{\mathrm{t}}, K_{2}, \psi_{\delta}, I_{\mathrm{r}}, \alpha, \Delta p, n, V\right)
\end{aligned}
$$


在工况切换过程中, 变量百能在较短时间内达到 目标排量并稳定，可忽略泵加速过程的能量损失 ${ }^{[6]}$ 。 由式(13)知, 当电动机加速度 $\alpha$ 不等于 0 时, 视为加(减) 速过程; 当电动机加速度 $\alpha$ 等于 0 时, 视为匀速过程。 驱动单元加速过程的能量损失 $E_{\alpha}$ 和勺速过程的能量损 失 $E_{\mathrm{n}}$ 分别如式(14)和式(15)所示

$$
\begin{aligned}
& E_{\alpha}=\int_{0}^{t_{\mathrm{a}}} \varphi\left(M_{\mathrm{L}}, J_{\mathrm{t}}, K_{2}, \psi_{\delta}, I_{\mathrm{r}}, \alpha, \Delta p, n, V\right) \mathrm{d} t \quad \alpha \neq 0 \\
& E_{n}=\int_{0}^{t_{\mathrm{b}}} \varphi\left(M_{\mathrm{L}}, J_{\mathrm{t}}, K_{2}, \psi_{\delta}, I_{\mathrm{r}}, \Delta p, n, V\right) \mathrm{d} t \quad \alpha \neq 0
\end{aligned}
$$

式中, $t_{\mathrm{a}}$ 为加(减)速时间; $t_{\mathrm{b}}$ 为匀速时间。整个动态 过程的能量损失为

$$
\begin{aligned}
& E_{\text {loss }}=E_{\alpha}+E_{n}= \\
& \int_{0}^{t_{\mathrm{a}}} \varphi\left(M_{\mathrm{L}}, J_{\mathrm{t}}, K_{2}, \psi_{\delta}, I_{\mathrm{r}}, \alpha, \Delta p, n, V\right) \mathrm{d} t+ \\
& \int_{t_{\mathrm{a}}}^{t} \varphi\left(M_{\mathrm{L}}, J_{\mathrm{t}}, K_{2}, \psi_{\delta}, I_{\mathrm{r}}, \Delta p, n, V\right) \mathrm{d} t
\end{aligned}
$$

当一组电动机转速和泵排量组合切换至另一组 电动机转速和泵排量组合时, 可视为某一具体的切 换过程。其中 $\Delta p$ 由系统运行特性确定, 可认为其和 静态工况下的 $n 、 V$ 均为定值 ${ }^{[8]}$ 。因此, 在某一具体 的切换过程中, 可认为系统的 $\Delta p 、 n$ 和 $V$ 的变化与 电动机加速度无关, 故可忽略它们对不同加速度下 动态过程能量损失的影响。结合式(16)知, 影响变 速变排量液压驱动系统动态过程能量损失的因素包 括电动机加速度 $\alpha$ 和动态过程的时间 $t\left(t=t_{\mathrm{a}}+t_{\mathrm{b}}\right)$ 。 通过合理调整电动机加速度 $\alpha$ 和动态过程的时间 $t$, 可减少动态过程能量损失 $E_{\text {loss }}$, 结合式(17)知, 即可 提高整个动态过程的能效 $\eta$ 。

$$
\eta=\frac{E_{\text {in }}-E_{\text {loss }}}{E_{\text {in }}}=1-\frac{E_{\text {loss }}}{E_{\text {in }}}
$$

式中, $E_{\text {in }}$ 为动态过程的输入能耗, 针对某一具体的 切换过程, 其值视为恒定。

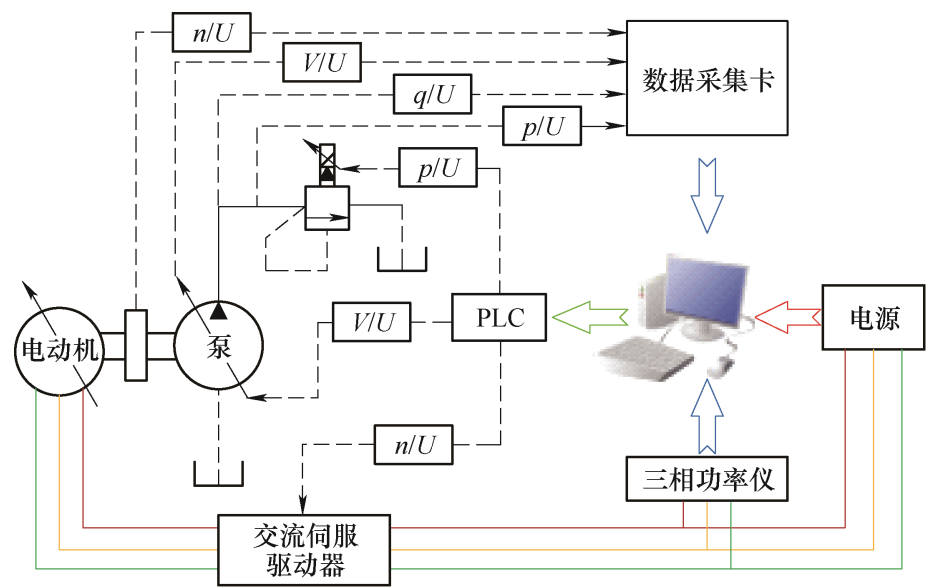

(a) 原理图
对于动态过程, 系统的稳定性是前提, 将电动 机转速波动 $\Delta n$ 作为衡量系统稳定性的指标 ${ }^{[13]}$ 。根据 系统稳定性要求设置系统所允许的最大转速波动为 $\Delta n_{\max }$, 在保证实际最大转速波动 $\Delta n_{\mathrm{a}} \leqslant \Delta n_{\max }$ 前提下, 降低动态过程的能量损失。故采用如下优化模型

$$
\begin{array}{ll}
\min & E_{\text {loss }} \\
\text { s.t. } & \Delta n_{\mathrm{a}} \leqslant \Delta n_{\max } \\
& 0 \leqslant \alpha \leqslant \alpha_{\max } \\
& 0 \leqslant t \leqslant t_{\max }
\end{array}
$$

式中, 实际最大转速波动 $\Delta n_{\mathrm{a}}$ 为整个动态过程最大 (小)转速与稳定转速之差的绝对值。

由式(18)知, 先根据系统稳定性要求设置 $\Delta n_{\max }$, 在保证 $\Delta n_{\mathrm{a}} \leqslant \Delta n_{\text {max }}$ 的前提下，通过调整电动机加速 度 $\alpha$ 和 $t$ 可得到动态过程的最小能量损失对应下的 电动机加速度 $\alpha$, 即完成动态过程的优化。

\section{2 变速变排量驱动系统动态能耗结 果与讨论}

\section{1 试验平台}

为进一步探究变速变排量液压驱动系统动态过 程的能量损失与加速度的关系，搭建伺服电动机驱动 比例变量洜组成的液压驱动单元试验平台, 其原理图 $3 \mathrm{a}$ 和实物图 $3 b$ 如下所示。液压试验台包括油箱、比 例变量泵(A37-F-R-04E-16M-60-42, 日本油研)、伺服 电动机(SMH180D-0350-15AAk-4HKC，步进科技)、 电磁比例溢流阀(TDBET6E，山东泰丰)。利用运行在 上位机的 LabVIEW 建立与 PLC(S7-200SMART, 西 门子)的通信, 从而控制变量洜排量和系统压力。通 过 PLC 控制伺服驱动器(JD630-LA-000, 步进科技) 从而控制电动机速度，伺服电动机、比例变量百和电 磁比例溢流阀主要参数见表 1 。

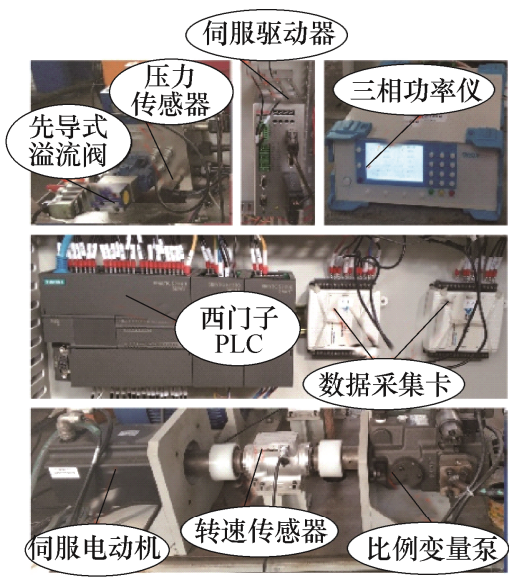

(b) 实物图

图 3 伺服电动机驱动比例变量原试验台 
表 1 伺服电动机、比例变量洜和先导式比例溢流阀主要参数

\begin{tabular}{|c|c|c|c|c|c|c|}
\hline \multicolumn{3}{|c|}{ 伺服电动机 } & \multicolumn{3}{|c|}{ 比例变量泵 } & \multirow{2}{*}{$\frac{\text { 溢流阀 }}{\text { 压力 } p_{\max } / \mathrm{MPa}}$} \\
\hline $\begin{array}{c}\text { 功率 } \\
P_{\mathrm{N}} / \mathrm{kW} \\
\end{array}$ & $\begin{array}{c}\text { 加速度 } \\
\alpha_{\max } /\left(\mathrm{rad} / \mathrm{s}^{2}\right) \\
\end{array}$ & $\begin{array}{c}\text { 转速 } \\
n_{\max } /(\mathrm{r} / \mathrm{min})\end{array}$ & 压力 $p_{\max } / \mathrm{MPa}$ & $\begin{array}{c}\text { 排量 } \\
V_{\max } /(\mathrm{mL} / \mathrm{r})\end{array}$ & 转速 $n_{\min } \sim n_{\max } /(\mathrm{r} / \mathrm{min})$ & \\
\hline 3.5 & 2000 & 1500 & 21 & 36.9 & $600 \sim 1800$ & 31.5 \\
\hline
\end{tabular}

系统的实时输入功率 $P_{\text {in }}$ 通过三相功率分析 仪(AWS2103, 爱特) 获取, 并通过 Modbus-RTU 协议传入上位机。电动机的实时转速 $n$ 、泵的实 时排量 $V$ 、驱动系统的输出流量 $q$ 和压力值 $p_{\text {out }}$ 分别采用转矩转速传感器 (ZH07, 北京中航)、变 量洜自带的排量传感器、流量传感器 (FH-LWGY-15GA, 合肥丰杭) 和压力传感器 (KS-N-E-E-B25D-M-V, 意大利杰佛伦)通过数据 采集卡(USB-6009，NI)转换后传入上位机。

假定油箱液压油的压力为零, 则泵进出口压力 差 $\Delta p$ 与泵出口压力 $p_{\text {out }}$ 相等, 系统的实际输出功率 $P_{\text {out }}$ 可由式(19)得出。

$$
P_{\text {out }}=\Delta p \cdot q=p_{\text {out }} \cdot q
$$

式中, $q$ 为驱动系统输出流量。

假定动态过程的时间是 $t_{1}$ 到 $t_{2}$, 则驱动系统动 态过程的实际能量损失可由式(20)得出。

$$
E_{\text {loss }}=E_{\text {in }}-E_{\text {out }}=\int_{t_{1}}^{t_{2}} P_{\text {in }} \mathrm{d} t-\int_{t_{1}}^{t_{2}} p_{\text {out }} \cdot q \mathrm{~d} t
$$

为模拟变速变排量液压驱动系统的实际动态过 程, 设置 3 组电动机转速和永排量的切换过程, 其 参数设定见表 2 。

\section{表 2 各切换过程电动机转速和洜排量参数设置}

\begin{tabular}{ccc}
\hline 阶段 & 电动机转速 $/(\mathrm{r} / \mathrm{min})$ & 泵排量 $/(\mathrm{mL} / \mathrm{r})$ \\
\hline 初始参数 & 600 & 14.0 \\
目标参数 1 & 1050 & 8.5 \\
目标参数 2 & 1450 & 7.0 \\
目标参数 3 & 950 & 6.5 \\
\hline
\end{tabular}

按照动态过程分析, 设置三组具体的切换过 程为: $D_{1}$ : 初始参数向目标参数 1 切换; $D_{2}$ : 目 标参数 1 向目标参数 2 切换; $D_{3}$ : 目标参数 2 向 目标参数 3 切换。对于任意具体的切换过程, 通 过调整电动机加速度从 $0 \mathrm{rad} / \mathrm{s}^{2}$ 至 $2000 \mathrm{rad} / \mathrm{s}^{2}$ 变 化, 均可由式(20)得出该切换过程不同加速度下 的能量损失 $E_{\text {loss }}$ 。

\section{2 动态能量损失与电动机加速度的关系}

在某一具体切换过程中, 以液压缸推杆运行相 同位移为标准，保证切换过程液压缸推杆所做有用
功相同。相对于电动机, 变量泵的动态性能较佳, 故忽略原排量影响 ${ }^{[6]}$ 。易得液压缸推杆的位移与电 动机角位移成正相关。

电动机角位移 $s$ 与加速度 $\alpha$ 的关系满足式(21)

$$
s=\frac{n_{\mathrm{t}}^{2}-n_{0}^{2}}{2 \alpha}
$$

式中, $n_{0}$ 和 $n_{\mathrm{t}}$ 分别表示某一具体切换过程中电动机 的始、末速度。

由式(21)可知, 当电动机加速度趋于最小值时, 相对于其他加速度, 最小加速度(本试验最小加速度 设置 $\alpha_{\mathrm{min}}=5 \mathrm{rad} / \mathrm{s}^{2}$ )对应的角位移最大。为保证动态 过程电动机各加速度下的角位移相同, 设置电动机 最小加速度对应的最大角位移 $s_{\max }$ 作为某一具体切 换过程中液压缸推杆所做有用功相同的基准位移。 假定电动机在某一具体切换过程所有加速度下均达 到 $s_{\text {max }}$ 时, 则最小加速度下的切换过程只存在加(减) 速过程, 而其他加速度下的切换过程均存在加(减) 速过程和匀速过程。

由第 1.2 节可知, 影响加(减)速过程能量损失 $E_{\alpha}$ 的因素主要是驱动器的功率损失 $P_{\mathrm{c}}$ 和电动机的定 子铜损 $P_{\mathrm{Cus}}$ 对于伺服电动机, 转子是永磁体, 其损 耗主要为磁滞损耗和浴流损耗, 一般忽略不计 ${ }^{[18]}$ )。 功率损失 $P_{\mathrm{c}} 、 P_{\mathrm{Cus}}$ 与电动机加速度的关系如式(22)。

$$
P_{\mathrm{c}} \propto \alpha, P_{\text {Cus }} \propto k+\alpha^{2}
$$

式中, $k$ 为常量。

假定加(减)速过程电动机转速从 $n_{0}$ 变化到 $n_{\mathrm{t}}$, 时间为 $t_{\mathrm{a}}$, 加(减)速过程能量损失 $E_{\alpha}$ 与电动机加速 度 $\alpha$ 满足式(23)

$$
\begin{aligned}
& E_{\alpha} \propto=P_{\mathrm{c}}+P_{\text {Cus }}=\left(k+\alpha+\alpha^{2}\right) \cdot t_{\mathrm{a}}= \\
& \left(k+\alpha+\alpha^{2}\right) \cdot\left(\frac{n_{\mathrm{t}}-n_{0}}{\alpha}\right)= \\
& \left(n_{\mathrm{t}}-n_{0}\right) \cdot\left(\frac{k}{\alpha}+1+\alpha\right)
\end{aligned}
$$

由式(23)可知, $E_{\alpha}$ 随 $\alpha$ 的增大呈先急剧减小后增大 的趋势。

由试验分析得到加(减)速过程能量损失 $E_{\alpha}$ 与电 动机加速度 $\alpha$ 关系如图 4 所示, $E_{\alpha}$ 随 $\alpha$ 的增大呈先 减小再增大的趋势。 


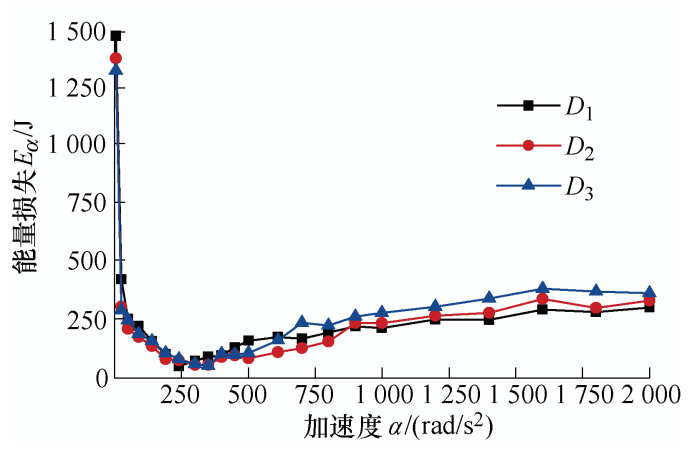

图 4 三组不同动态加速过程 $E_{\alpha}-\alpha$ 曲线

对于匀速过程, 式(15)中 $K=\varphi\left(M_{\mathrm{L}}, K_{2}, \psi_{\delta}, \Delta p, n, V\right)$ 为定值, 匀速过程的能量损失 $E_{\mathrm{n}}$ 与只与匀速过程的 时间 $t_{\mathrm{b}}$ 有关，其中 $E_{\mathrm{n}}$ 满足式(24)

$$
E_{\mathrm{n}}=K \cdot t_{b}=K \cdot \frac{s_{\text {max }}-\frac{n_{t}^{2}-n_{0}^{2}}{2 \alpha}}{n_{t}}
$$

由式(24)可知, $E_{\mathrm{n}}$ 随电动机加速度 $\alpha$ 的增大呈先增 大后稳定的趋势。

由试验分析得到匀速过程能量损失 $E_{\mathrm{n}}$ 与电动 机的加速度 $\alpha$ 关系如图 5 所示, $E_{\mathrm{n}}$ 随 $\alpha$ 的增大呈先 急剧增大后稳定的趋势。

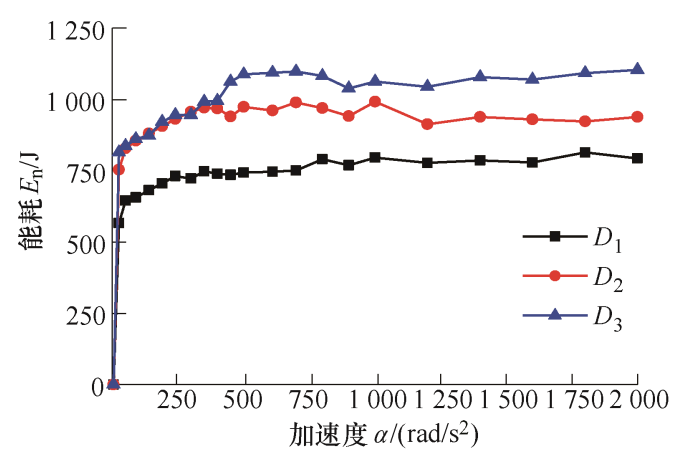

图 5 三组不同动态匀速过程 $E_{\mathrm{n}}-\alpha$ 曲线

联立式(23)和(24)得到任意具体切换过程的能 量损失 $E_{\text {loss }}$ 如式(25)所示

$$
\begin{gathered}
E_{\text {loss }}=E_{\alpha}+E_{\mathrm{n}} \propto \frac{k_{1}}{\alpha}+k_{2} \alpha+k_{3} \\
k_{1}=\left(n_{\mathrm{t}}-n_{0}\right)\left[2 k n_{\mathrm{t}}-K\left(n_{\mathrm{t}}+n_{0}\right)\right] / 2 \alpha n_{\mathrm{t}} \\
k_{2}=n_{\mathrm{t}}-n_{0} \quad k_{3}=\left(n_{\mathrm{t}}-n_{0}\right) / k+K s_{\max } /\left(n_{\mathrm{t}} k\right)
\end{gathered}
$$

由式(25)得, 对于任意具体切换过程能量损失随电 动机加速度的增大呈先减小后增大的趋势。

任意具体切换过程的能量损失 $E_{\mathrm{loss}}$ 包括加速过 程能量损失 $E_{\alpha}$ 和匀速过程的能量损失 $E_{\mathrm{n}}$, 由试验分 析得到 $E_{\text {loss }}$ 与电动机加速度 $\alpha$ 的关系如图 6 所示, $E_{\text {loss }}$ 随 $\alpha$ 的增大呈先减小后增大的趋势。

由上述理论与试验对照分析可知, 在任意工况 切换过程中, 存在最佳加速度使整个切换过程的能 量损失最小。故工况切换过程能效优化的目的是得 出切换过程最低能量损失的电动机加速度 $\alpha_{\mathrm{opt}}$ 。

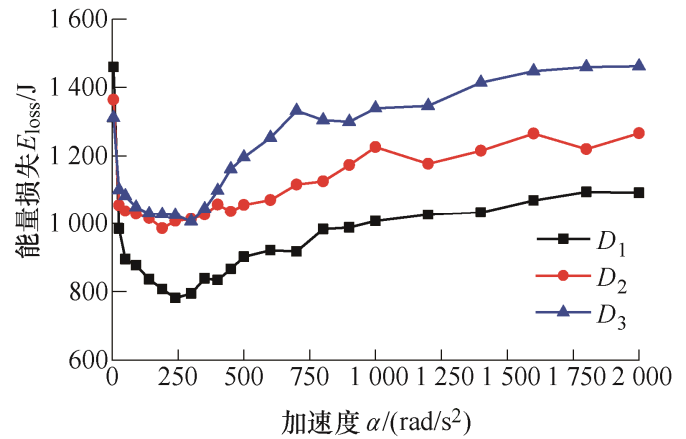

图 6 三组具体切换过程 $E_{\mathrm{loss}}-\alpha$ 曲线

\section{3 动态过程稳定性分析}

由图 6 知, 阴影部分对应的电动机加速度区间 为 $\left(125 \mathrm{rad} / \mathrm{s}^{2} ， 375 \mathrm{rad} / \mathrm{s}^{2}\right)$ 。当电动机加速度 $\alpha=150 \mathrm{rad} / \mathrm{s}^{2}$ 时, 动态切换过程 $n-t$ 和 $V-t$ 曲线分别 如图 7a、7b 所示。针对变速变排量液压驱动系统的 动态过程, 电动机的转速波动将会造成流量脉动并 影响系统的稳定性 ${ }^{[12]}$ 。对于试验所设置的伺服电动 机驱动比例变量泵系统, 系统输出流量是电动机和 泵共同作用的结果。在电动机加速度 $\alpha=150 \mathrm{rad} / \mathrm{s}^{2}$ 时的动态过程中, 如图 7a 所示, 电动机转速存在较 大的波动, 且存在较长的调整时间(在某一工况切换 过程中, 电动机转速首次达到目标值 $\pm 2 \%$ 时的对应 时间与其开始变化时的对应时间之差); 如图 $7 \mathrm{~b}$ 所 示, 泵的动态特性较佳, 在考虑驱动系统动态过程 时, 忽略其对动态过程系统稳定性的影响。

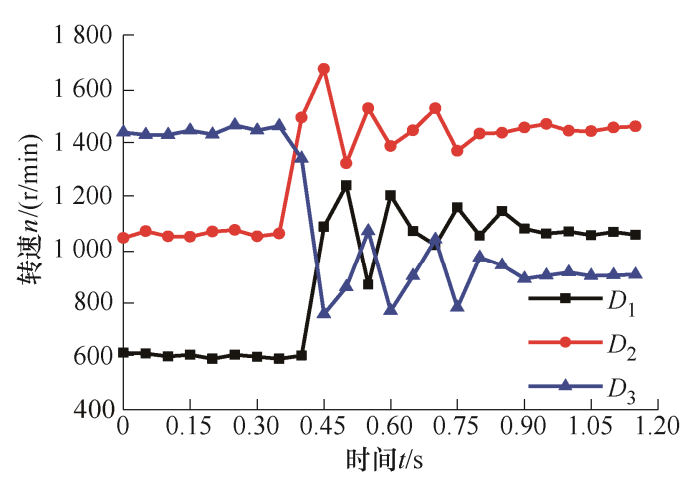

(a) 转速

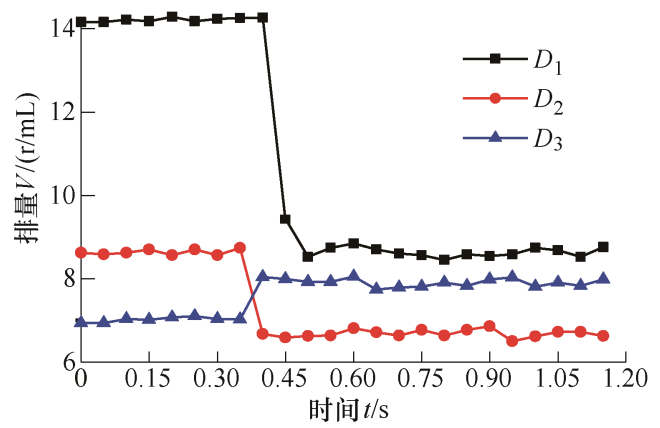

(b) 排量

图 7 伺服电动机 $\alpha=150 \mathrm{rad} / \mathrm{s}^{2}$ 时三组切换过程和曲线 
在第 2.1 节设置的三组具体切换过程 $\left(D_{1} 、 D_{2}\right.$ 、 $\left.D_{3}\right)$ 中, 不同电动机加速度 $\alpha$ 与转速波动量 $\Delta n(\Delta n$ 表 示转速最大波动量与目标转速之差的绝对值)、调整
时间 $t_{\mathrm{s}}$ 之间的关系见表 3 。由表 3 知, 电动机转速 波动 $\Delta n$ 随着电动机加速度 $\alpha$ 的增大而增大, 且加速 度越大, 调整时间 $t_{\mathrm{s}}$ 越长。

表 3 三组切换过程不同电动机加速度下时间 $t_{\mathrm{s}}$ 和 $\Delta n$ 关系对照表

\begin{tabular}{|c|c|c|c|c|c|c|c|}
\hline & $\alpha /\left(\mathrm{rad} / \mathrm{s}^{2}\right)$ & 25 & 100 & 300 & 1000 & 1500 & 2000 \\
\hline \multirow{2}{*}{$D_{1}$} & 时间 $t_{\mathrm{s} 1} / \mathrm{s}$ & 0.5 & 0.65 & 0.8 & 0.95 & 1.15 & 1.35 \\
\hline & $\begin{array}{c}\text { 转速波动量 } \\
\Delta n_{1} /(\mathrm{r} / \mathrm{min})\end{array}$ & 22.10 & 136.63 & 350.96 & 429.34 & 485.13 & 470.63 \\
\hline \multirow[b]{2}{*}{$D_{2}$} & 时间 $t_{\mathrm{s} 2} / \mathrm{s}$ & 0.5 & 0.6 & 0.75 & 0.9 & 1.1 & 1.25 \\
\hline & $\begin{array}{c}\text { 转速波动量 } \\
\Delta n_{2} /(\mathrm{r} / \mathrm{min})\end{array}$ & 21.51 & 136.184 & 182.27 & 236.96 & 280.65 & 327.58 \\
\hline \multirow{2}{*}{$D_{3}$} & 时间 $t_{\mathrm{s} 3} / \mathrm{s}$ & 0.55 & 0.75 & 0.85 & 0.95 & 1.15 & 1.3 \\
\hline & $\begin{array}{c}\text { 转速波动量 } \\
\Delta n_{3} /(\mathrm{r} / \mathrm{min})\end{array}$ & 25.07 & 86.93 & 270.64 & 397.86 & 436.60 & 445.17 \\
\hline
\end{tabular}

电动机转速波动导致流量波动, 流量的波动会 导致液压缸推杆振动并影响系统的稳定性 ${ }^{[14]}$ 。因此 液压缸推杆的振动与电动机转速的波动有关, 可通 过调整电动机加速度对电动机转速波动进行限制从 而保证系统稳定性。

\section{3 应用实例}

为分析液压驱动系统动态能耗优化效果, 以圆 筒拉深成形过程为例, 分析优化后系统动态过程的 能效。液压系统成形过程主要包括快降(FF)、压制 $(\mathrm{PF}) 、$ 保压 $(\mathrm{PM}) 、$ 卸荷 $(\mathrm{UD}) 、$ 快回(FR)和慢回(SR)6 个动作, 其中快降 $(\mathrm{FF}) 、$ 压制 $(\mathrm{PF}) 、$ 快回(FR)和慢回 $(\mathrm{SR})$ 是成形过程所必需存在的, 其他过程则根据工 艺需求确定是否保留 ${ }^{[5]}$ 。位移、需求压力和需求流 量工艺曲线如图 8 所示, 各工况的压力、转速和排 量见如表 4 所示。

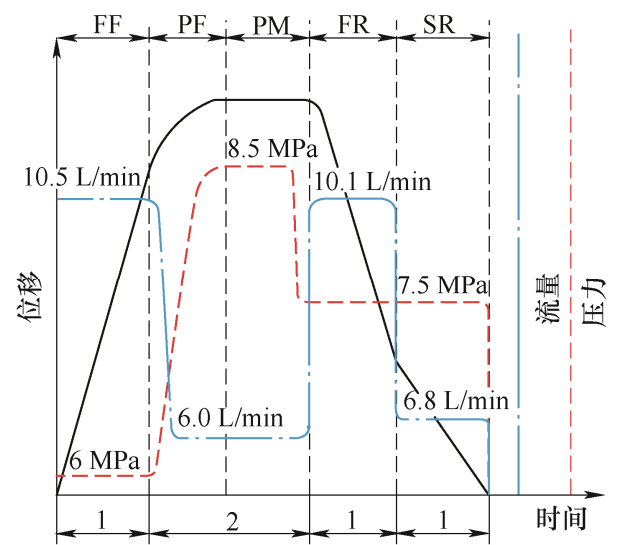

图 8 滑块的位移、需求压力和需求流量工艺曲线
表 4 液压成形过程各工况工艺参数

\begin{tabular}{cccc}
\hline \multicolumn{2}{c}{ 工况 } & 电动机转速 $/(\mathrm{r} / \mathrm{min})$ & 恶排量 $/(\mathrm{mL} / \mathrm{r})$ \\
\hline $\mathrm{FF}$ & 工况 1 & 1050 & 10.0 \\
$\mathrm{PF}, \mathrm{PM}$ & 工况 2 & 700 & 8.5 \\
$\mathrm{FR}$ & 工况 3 & 1450 & 7.0 \\
$\mathrm{SR}$ & 工况 4 & 900 & 7.5 \\
\hline
\end{tabular}

在液压成形过程中, 由上述分析并结合式(26) 可知, 电动机加速度过大导致液压缸推杆振动, 并 易造成液压滑块振动。对于圆筒的拉伸成形过程而 言, 首先应保证系统的稳定性, 防止滑块振动。在 工况切换过程中, 逐渐增大电动机加速度并利用位 移传感器采集滑块在稳定工况(流量无波动)下的实 时位移 $s$, 当 $\mathrm{d}^{2} s / \mathrm{d} t^{2} \neq 0$ 时, 记录此时的电动机加速 度 $\alpha_{\max }$ 。为防止滑块产生振动而造成的系统不稳定, 取成形过程的电动机加速度 $\alpha<\alpha_{\text {max }}$ 。对于圆筒拉深 成形的压制(PF)过程, 由式(27)知 ${ }^{[15]}$, 根据已知工艺 参数(极限拉深比 $L D R$ 、模具与板料实际接触面积 $A^{*}$ 、 模具尺寸 $d_{\mathrm{p}}$ 、板料厚度 $t$ ) 求得滑块的极限速度 $v_{\text {max }}$, 以保证工件的成形质量。根据极限速度 $v_{\text {max }}$ 并结合式 (26)求得系统所允许的最大流量波动 $Q_{\max }$ 以确定电动 机最大转速 $n_{\max }$, 结合试验得出的转速波动 $\Delta n$ 与电动 机加速度 $\alpha$ 的对照表, 选择出合理的电动机加速度以 保证工件的成形质量和系统稳定性。按上述分析, 该 案例中保证电动机加速度 $\alpha \leqslant 500 \mathrm{rad} / \mathrm{s}^{2}$ 。

$$
\begin{gathered}
\ln L D R<0.74-\frac{3 A_{\mathrm{r}}^{*}+0.011118 P(v-0.1)}{\pi d_{p}^{t}} \\
P=534888.9 A_{\mathrm{r}}^{* 0.85}-437657.4 A_{\mathrm{r}}^{* 0.3}
\end{gathered}
$$

该案例的拉深成形过程中, 相邻工况切换形成 三组具体的切换过程 $d_{1} 、 d_{2}$ 和 $d_{3}$ 。在保证工件成形 质量和系统稳定性的前提下, 调整电动机加速度, 
由式(20)获得 $d_{1} 、 d_{2}$ 和 $d_{3}$ 不同加速度对应下的能量 损失。对比任一具体的切换过程不同加速度对应 下的能量损失, 得出该切换过程最低能量损失对 应的电动机最佳加速度。进而得出: 三组具体切 换过程电动机采用默认加速度 $\left(\alpha=610 \mathrm{rad} / \mathrm{s}^{2}\right)$ 对应 的输入能耗 $E_{\mathrm{in}}$ 、能量损失 $E_{\mathrm{def}}$ 和电动机转速波动
量 $\Delta n_{\mathrm{def}}$; 三组具体切换过程的电动机最佳加速度 对应的能量损失 $E_{\min }$ 和转速波动量 $\Delta n_{\min }$; 使用最 佳加速度比默认加速度节省的能量 $\Delta E_{\text {loss }} ; \Delta E_{\text {loss }}$ 与 $E_{\text {in }}$ 之比 $\lambda$; 降低的转速波动量 $\Delta n_{\text {def }}-\Delta n_{\text {min }}$ 与采 用默认加速度时电动机转速波动量 $\Delta n_{\text {def }}$ 之比 $\delta$ 。 其对应关系如表 5 所示。

表 5 能量损失 $\left(E_{\mathrm{def}}\right) 、$ 动态能量损失 $\left(\Delta E_{\mathrm{loss}}\right)$ 、能耗比 $(\lambda)$ 关系对照表

\begin{tabular}{|c|c|c|c|c|c|c|c|c|}
\hline & 能量 $E_{\mathrm{in}} / \mathrm{J}$ & 能量 $E_{\mathrm{def}} / \mathrm{J}$ & 能量 $E_{\min } / \mathrm{J}$ & 能量 $\Delta E_{\mathrm{loss}} / \mathrm{J}$ & 能耗比 $\lambda(\%)$ & 转速 $\Delta n_{\mathrm{def}} /(\mathrm{r} / \mathrm{min})$ & 转速 $\Delta n_{\min } /(\mathrm{r} / \mathrm{min})$ & 能效 $\delta(\%)$ \\
\hline$d_{1}$ & 2827.9 & 905.1 & 712.6 & 192.5 & 6.8 & 384.3 & 341.4 & 11.1 \\
\hline$d_{2}$ & 3166.3 & 1121.5 & 874.3 & 247.2 & 7.8 & 236.0 & 192.6 & 18.5 \\
\hline$d_{3}$ & 2112.0 & 963.4 & 765.1 & 198.3 & 9.3 & 342.4 & 290.2 & 15.1 \\
\hline
\end{tabular}

由表 5 可知, 在拉深成形的三组具体切换过程 中, 与使用默认加速度相比, 使用最佳电动机加速 度使整个动态过程的能效平均提高了 $8.0 \%$, 平均节 省能量 $221.1 \mathrm{~J}$, 转速波动量平均降低了 $14.9 \%$ 。假 定有 20 台此类液压机的车间工作 $8 \mathrm{~h}$, 在动态切换 过程中每天可节约 $26.5 \mathrm{~kW} \cdot \mathrm{h}$ 左右电量。

\section{4 结论}

(1) 介绍了变速变排量液压驱动系统的动态过 程, 建立了动态过程优化模型。根据优化模型得出 动态能量损失与电动机加速度的关系, 并结合试验 分析得到动态过程最低能量损失对应的电动机加 速度。

(2) 对变速变排量驱动系统动态过程的稳定性 进行了试验分析, 得出液压缸推杆振动与电动机转 速波动有关, 选择合理的电动机加速度以限制电动 机转速波动, 保证系统稳定性。

(3) 以液压拉深成形过程为例, 结合系统稳定 性和工艺要求提出一种电动机加速度的优化方法。 通过分析成形过程的动态能耗, 得出电动机采用最 佳电动机加速度比默认加速度使整个动态切换过程 的能效平均提高了 $8.0 \%$, 电动机转速波动量平均降 低了 $14.9 \%$ 。对于工况频繁切换的变速变排量液压 驱动系统, 研究其动态能耗具有一定意义。

\section{参 考 文 献}

[1] HO T H, AHN K K. Modeling and simulation of hydrostatic transmission system with energy regeneration using hydraulic accumulator[J]. Journal of Mechanical Science and Technology, 2010, 24(5): 1163-1175.

[2] WANG Tao, WANG Qingfeng, LIN Tianliang. Improvement of boom control performance for hybrid hydraulic excavator with potential energy recovery[J]. Automation in Construction, 2013, 30: 161-169.

[3] KWON T S, LEE S W, SUL S K, et al. Power control algorithm for hybrid excavator with supercapacitor [J]. IEEE Transactions on Industry Applications, 2008, 46(4): 1447-55.

[4] LI Lei, HUANG Haihong, ZHAO Fu, et al. Analysis of a novel energy-efficient system with double-actuator for hydraulic press [J]. Mechatronics, 2017, 47: 77-87.

[5] LI Lei, HUANG Haihong, LIU Zhifeng, et al. An energy-saving method to solve the mismatch between installed and demanded power in hydraulic press[J]. Journal of Cleaner Production, 2016, 139: 636-45.

[6] GE Lei, QUAN Long, ZHANG Xiaogang, et al. Power matching and energy efciency improvement of hydraulic excavator driven with speed and displacement variable power source[J]. Chinese Journal of Mechanical Engineering, 2019, 32(1): 100.

[7] SHI Hu, YANG Huayong, GONG Guofang, et al. Energy saving of cutterhead hydraulic drive system of shield tunneling machine $[\mathrm{J}]$. Automation in Construction, 2014, 37(1): 11-21.

[8] HUANG Haihong, JIN Rui, LI Lei, et al. Improving the energy efficiency of a hydraulic press via variable-speed variable-displacement pump unit[J]. Journal of Dynamic Systems, Measurement, and Control, 2018, 140(11).

[9] WILLKOMM J, WAHLER M, WEBER J. Quadratic programming to optimize energy efficiency of speed-and displacement-variable pumps[C]// 8th FPNI Ph.D. Symposium on Fluid Power, Lappeenranta, Finland. June 11-13, 2014.

[10] 李泽鹏, 权龙, 葛䂞, 等. 液电混合驱动液压挖掘机动 臂特性及能效研究 [J]. 机械工程学报, 2018, 54(20): 235-241.

LI Zepeng, QUAN Long, GE Lei, et al. Research on 
characteristics and energy efficiency of hydraulic-electric combined driving hydraulic excavator boom[J]. Journal of Mechanical Engineering,2018, 54(20): 235-241.

[11] SUN Hui, JING Junqing. Research on the system configuration and energy control strategy for parallel hydraulic hybrid loader[J]. Automation in Construction, 2010, 19(2): 213-220.

[12] 间政, 权龙. 变转速变排量双控轴向柱塞泵脉动特性及 噪声的研究 [J]. 机械工程学报, 2016, 52(16): 176-184. YAN Zheng, QUAN Long. Characteristics of pulsation and noise in the axial piston pump with displacement and speed compound control[J]. Journal of Mechanical Engineering, 2016, 52(16): 176-184.

[13] 胡东明. 液压电梯变转速闭式电液系统速度控制特性 研究 [D]. 杭州: 浙江大学, 2009.

HU Dongming. Research on speed control characteris-tics of hydraulic elevator variable speed closed electrohy-draulic system [D]. Hangzhou : Zhejiang University, 2009.

[14] 刘艳. 液压机冲裁主动减振技术研究[D]. 广州: 广东工 业大学, 2008.

LIU Yan. Research on active vibration reducing method of blanking[D]. Guangzhou : Guangdong University of Technology, 2008.

[15] 付秀娟. 变速/变载条件下板料冲压成形性能及其变形
机理研究[D]. 武汉：华中科技大学, 2012.

FU Xiujuan. A dissertation submitted in partial fulfillment of the requirements for the degree of doctor of philosophy in engineering[D]. Wuhan: Huazhong University of Science \&Technology, 2012.

[16] GUO Qing, QIANG Wang, LI Xiaochai. Finite-time convergent control of electrohydraulic velocity servo system under uncertain parameter and external load[J]. IEEE Transactions on Industrial Electronics, 2018, 66(6): 4513-4523.

[17] BAI Baodong, CHEN Dezhi. Inverter IGBT loss analysis and calculation[C]//2013 IEEE International Conference on Industrial Technology (ICIT). IEEE, Cape Town, South Africa 2013: 563-569.

[18] CAVALLARO C, DITOMMASO A O, MICELI R, et al. Efficiency enhancement of permanent-magnet synchronous motor drives by online loss minimization approaches[J]. IEEE Transactions on Industrial Electronics, 2005, 52(4): $1153-1160$

作者简介: 黄海鸿(通信作者), 男, 1980 年出生, 博士, 教授, 博士研 究生导师。主要研究方向为绿色制造、再制造与回收再资源化等。

E-mail: huanghaihong@hfut.edu.cn

唐运先, 男, 1994 年出生, 硕士研究生。主要研究方向为机电系统绿色 设计。

E-mail: tangyunxian1234@163.com 